\title{
Genital warts in children: what do they mean?
}

\author{
Y Jayasinghe, S M Garland
}

Arch Dis Child 2006;91:696-700. doi: 10.1136/adc.2005.092080

Human papillomaviruses (HPVs) are a diverse family of viruses, of which 30-40 genotypes specifically infect the genital tract. Genital HPVs are largely transmitted sexually, with most infections being asymptomatic and transient. In contrast, persistent infection with oncogenic genotypes in a minority is a strong risk factor, for subsequent development of high grade dysplasia, the precursor lesion to cervical neoplasia, which generally occurs after a long latency period. It is unknown whether there is a disease correlate in children chronically infected with oncogenic HPVs. Low risk HPV genotypes 6 and 11 are the primary cause of condylomata acuminata, although in children non-genital genotypes are also found in a proportion, with the mode of transmission being either perinatal, horizontal, or sexual. The finding of asymptomatic HPV DNA in children, and correlation with live virus, infectivity, or disease is unclear. Long term follow up for children with anogenital warts is recommended, although there are no longitudinal studies available to clarify whether they are at risk of developing carcinoma in young adulthood.

See end of article for authors' affiliations

Correspondence to:

Correspondence
Prof. S Garland, Department of

Microbiology \& Infectious Diseases, Royal Women's Hospital, 132 Grattan Street, Carlton, Victoria, Australia 3052; suzanne. garland@rch.org.au

Accepted 22 April 2006

Published Online First

2 May 2006

.................... have varied from $0 \%$ to $80 \% .^{3}{ }^{4}$ Of sexually abused children, HPV DNA, and/or abnormal Pap cytology has been reported in 3.4-33\%, and EGWs only in $0.3-2 \% .{ }^{4}$ Evaluation is complex, as most children who have been sexually abused will not show carriage of the virus, nor will have evidence of physical trauma. ${ }^{67}$

While sexual abuse needs to be considered in every case of EGWs, particularly in those over 2-3 years of age, HPV may be transmitted in other ways. The presence of warts or HPV DNA alone without supporting social and clinical information is not diagnostic of sexual abuse. An epidemiological study of 124 children with clinical HPV infection concluded that many children over 2 years of age acquired HPV from non-sexual contact. The positive predictive value of the presence of EGWs for sexual abuse was $37 \%$ for children aged 2-12 years, and increased with age $(70 \% \text { for children over } 8 \text { years of age. })^{8}$

The average incubation period from sexual HPV exposure to development of EGWs in adolescents and adults is about 3 months (range 3 weeks to 8 months), ${ }^{9}$ but is unknown in children.

\section{Perinatal transmission}

Perinatal transmission is thought to largely occur via the intrapartum route, from direct contact with an infected maternal genital tract. HPV DNA has been reputed to be transmitted transplacentally. HPV DNA has been found in products of conception from spontaneous abortions, but this could represent contamination as the products pass through the maternal genital tract. ${ }^{10}$ In pregnant women with cervical dysplasia, HPV DNA has been found in amniotic fluid obtained by transabdominal amniocentesis prior to membrane rupture. ${ }^{11}$ Anal condylomata have been found in an infant at birth. ${ }^{12}$ The mother had recognised labial condylomata acuminata intrapartum and placental membranes had ruptured only 24 hours before vaginal breech delivery. HPV DNA has been reported in peripheral blood mononuclear cells (PBMCs) in those with urogenital infection, ${ }^{13}$ and HPV 16 DNA has been found in cord blood of babies whose mothers were positive for peripheral mononuclear cell DNA. ${ }^{14}$ Rather than transplacental transmission of infection, these findings can more adequately be explained by potential ascending infection via microscopic tears in the placental membranes, as occurs with other microorganisms. Viraemia has not been shown to occur with HPV, although HPV 16 DNA has been detected in banked frozen PBMC samples of paediatric HIV patients in the absence of sexual contact, and in samples of healthy donors. ${ }^{15}$

widely in the literature due to varying definitions of HPV infection (ranging from presence of HPV DNA to clinical lesions), ${ }^{2}$ the method of detection, and the population studied. In children with EGWs, reports of sexual abuse
Abbreviations: EGW, external genital wart; HPV, human papillomavirus 


\section{Case study}

A 4.5 year old girl was referred to the local child sexual assault service after recent disclosure of alleged digital fondling and penile-vaginal contact by an adult male carer. The last alleged episode of sexual assault was 3 months before consultation. Behavioural assessment was consistent with the child's history. Physical examination including genital inspection was normal, apart from two genital lesions consistent with condylomata acuminata.

The carer agreed to a clinical examination and was noted to have several penile condylomata acuminata.

A full STI screen (first void urine for Chlamydia trachomatis, Neisseria gonorrhoeae, and Trichomonas vaginalis, and blood tests for syphilis, hepatitis B virus, and HIV) was normal.

The child's mother was in a stable relationship, had no other partners, and no history of genital tract infections. A Pap smear taken antepartum showed low grade dysplasia, but this had regressed naturally to normal on her follow up Pap postpartum. The mother also suffered from recurrent finger warts.

Potential management issues arising from the case include:

- Had the child contracted infection from the mother; either from finger warts or genital HPV infection?

- Had the child contracted infection from the male caregiver: by sexual transmission or horizontal transmission?

- Is it possible to compare the viral types (DNA fingerprinting) to look for clonality and likely route of transmission?

- Could the child have picked up the virus from the male caregiver or the mother via fomites?

- Does the child have an increased risk of cervical dysplasia or ultimately cancer?

Earlier studies reported high rates of perinatal transmission of around $70 \%,{ }^{16} \quad 30 \%$ discordancy between HPV genotypes in mother and neonatal pairs, ${ }^{2}$ and high prevalence of HPV DNA in neonates of HPV negative women. HPV DNA contamination (in sample collection, transport, or within the laboratory) or transient colonisation of neonatal tissues from maternal colonisation may account for this. In a comprehensively conducted large study, 151 infants born to mothers with known HPV status were regularly evaluated until 3 years of age for the detection of HPV DNA using PCR in samples from the mouth, external genitalia, and anus. Results showed that the risk of perinatal transmission of HPVs, although present, is probably very low-at most $2.8 \% .{ }^{17}$ Moreover, progression from HPV colonisation to associated disease was uncommon. ${ }^{17}$ Some parallels can be drawn from mother to baby transmission with Chlamydia trachomatis. ${ }^{18} 19$

The upper age limit of perinatal infection is uncertain, and accepted to be as late as 5 years for laryngeal papillomatosis. ${ }^{8}$ Latent infection may occur, where there is no evidence of HPV clinically or by molecular diagnostics, but HPV is present in a non-productive state in cells remaining silent for years before clinical expression. ${ }^{4}$ This may explain the appearance of EGWs in immunocompromised patients who are either not sexually active or have no recent new sexual partners.

In a study of girls aged 2-11 years with lichen sclerosus, episomal (inactive) HPV DNA was detected by vulval scrapes and urine specimens in $25 \%$ of children whether they had vulval disease or not, in the absence of a history of sexual abuse. ${ }^{20}$ Nearly $50 \%$ of the mothers of children with lichen sclerosus had an abnormal smear within three years of the child's birth, and the authors concluded that vertical transmission had occurred in these patients.

Juvenile onset recurrent respiratory laryngeal papillomatosis (JORRP) due to HPV 6 and 11 is rare, occurring at an incidence of $0.3-1.0 / 100000 .^{2}$ A strong correlation exists between the HPV DNA type found in the infant buccal mucosa and that in the mother's cervical samples. Even then, only around half of mothers of infants with JORRP have a history of EGWs during pregnancy. The converse (the presence of maternal EGWs at delivery) fortunately has a low rate of transmission, at around 1 in $400 .^{21}$

\section{Autoinoculation or heteroinoculation}

Evidence exists for horizontal transmission of HPV DNA. ${ }^{22}$ Therefore, autoinoculation or heteroinoculation from nongenital warts may be a source of EGWs in children, although not proven. It has recently been recognised that the phylogenetically related mucosal HPV types 2, 27, and 57 are detected in cutaneous lesions, as well as oral and genital lesions. While HPV 2 was initially regarded as the HPV type causing common hand warts, with more sophisticated typing systems, type 27 is now realised to hold this role. HPV 27 is also commonly found as a non-genital HPV type in genital lesions of children, ${ }^{23}$ and along with HPV 3 (another common cutaneous wart virus), is found in $10-20 \%$ of EGWs of children in the absence of sexual abuse. ${ }^{19}$ HPV subtypes in children do not seem to show high degrees of site specificity (tropism) for either mucosal or cutaneous sites, as is seen in adults. ${ }^{3}$

HPV DNA has been found in finger brush samples of adults with genital HPV infection, raising the possibility that people with genital warts may transfer genital HPVs to their children by finger-genital contact. ${ }^{24}$ However, it is unknown if the presence of HPV DNA alone is capable of inducing active infection, particularly without abrasion of the skin.

\section{Fomites}

It has been postulated that HPV may be transmitted via fomites. Pao and colleagues ${ }^{25}$ found HPV DNA on vulval swab PCR in $14.8 \%$ of virginal girls. Another study of virginal girl relatives of adults with anogenital warts showed subclinical lesions in $75 \%$ (diagnosed by introital Pap cytology, vulvoscopy, and histology in 62\%.) The authors' criterion for exclusion of sexual abuse was an in-depth sexual history and gynaecological examination. ${ }^{26}$ Yet in four other studies of virginal young women, there were only two cases of HPV DNA found among a total of 289 women. ${ }^{27-30}$

It must be realised that detection of HPV DNA alone does not necessarily equate with live virus or for that matter infectivity, and does not have the same clinical or medicolegal significance as the finding of clinical lesions such as EGWs. The prevalence of HPV DNA using sensitive assays is high, yet clinical disease is uncommon, showing that the level of detection of HPV DNA by an individual assay (the analytical sensitivity) does not necessarily reflect a level of HPV DNA which is clinically relevant (clinical sensitivity). ${ }^{31}$ In other words, we are still trying to understand what a standalone positive HPV DNA test really means. Actively transcribed HPV infection may be tested for by checking for early region mRNA (E-mRNA), but results depend on the analytical sensitivity of the test; comparisons with clinical disease are awaited and this is largely a research tool. ${ }^{32}$

Therefore, although transmission by fomites is suggested by several studies, its impact in causing active infections and clinical disease is most likely small if at all. 
Is there a link with early onset anogenital neoplasia? EGWs are benign lesions due to low risk viral types, without oncogenic potential (with the exception of rare malignant change in Buschke-Lowenstein tumour). ${ }^{1}$ Anogenital cancers, however, are due to high risk types (16 and 18 accounting for $70 \%$ of all cases of cervical cancer worldwide). ${ }^{1}$ Chronic infection with oncogenic HPVs is a necessary risk factor for cervical neoplasia. There is generally a long latency between development of high risk HPV infection and cancer, and there are virtually no cancers reported before 19 years. ${ }^{33}$ In adults, EGWs may be a surrogate marker for co-existent carriage of high risk types of $\mathrm{HPV}^{34}$ or upper genital tract dysplasia. In children with EGWs, co-existent HPV 16 has been seen in cervicovaginal or intra-anal samples, although oncogenic potential in children is unclear. ${ }^{35}$ A preponderance of high risk subtypes has been detected in children with lichen sclerosus, but implications for cervical health are unknown. ${ }^{20}$ There has been one isolated report of bowenoid papulosis in an infant infected with HPV $16{ }^{36}$ While cervical dysplasia is more likely to resolve in adolescents compared to adults, recent studies indicate that cervical dysplasias in young women may be more common and progress faster than originally realised. ${ }^{37} 38$ It is unclear if this is due to early onset sexual activity (including childhood sexual abuse), or whether more intensive follow up has revealed that high grade dysplasia occurs earlier than originally considered. ${ }^{37-39}$ The HPV 16Ll vaccination has demonstrated high level protection against cervical dysplasia for at least 3.5 years after immunisation in adolescents aged $16-23$ years.$^{40}$ However, in children, until more is known about the likelihood of acquisition of high risk HPV types and their oncogenic risk, guidelines for Pap screening or HPV vaccine administration in children who have been sexually abused cannot be recommended.

\section{ROLE OF HPV GENOTYPING}

HPV DNA typing is a useful tool for cervical cancer prevention in adults, and there has been recent FDA approval in the United States for high risk HPV DNA screening in women over 30 years of age. In Australia, HPV DNA assays are largely research tools, apart from surveillance for those post-treatment for dysplasia. ${ }^{41}$

At present the evidence for performing HPV DNA typing in children and adolescents is not strong, and advised against because:

- HPV infection can be transient and the virus can be eliminated by a healthy immune system to undetectable levels. A negative test does not mean infection was not previously present.

- HPV DNA has been detected at various sites in nonabused children; other modes of transmission need to be considered, particularly mother to infant.

- Viral subtype alone cannot determine mode of transmission, because the virus does not display $100 \%$ tropism. $^{236}$ For example, HPV 6, classically a mucosatropic virus, occasionally can be found in lesions on the arms and legs. Furthermore, digital wart types may be transmitted sexually.

- HPV infection (particularly 6 and 11) in adults is widespread and often asymptomatic. The variability of the HPV 6 and 11 genomic sequences is not great; therefore circulating types may not vary between hosts, even if not directly transmitted from one to the other. Consequently sequencing two like viral genotypes from two individuals may not be able to prove transmission between the individuals.
- The presence of HPV DNA alone without visible disease or cytological abnormality does not warrant any change in medical management. ${ }^{6}$ There is no specific antiviral treatment for subclinical HPV infection. Some young women may undergo unnecessary follow up or treatment, ${ }^{38}$ as detection of minor cytologic abnormalities would be likely if Pap testing were performed on the basis of a positive HPV test. These abnormalities would most likely have resolved naturally.

- HPV testing is of limited value in women under 30 years due to the commonality of infection, and spontaneous resolution of infection in young women.

PLAN OF MANAGEMENT FOR A CHILD WITH EGWs A multidisciplinary, non-judgemental approach is recommended for children and their families. The following is recommended:

- A detailed history from the child and family, a thorough medical examination for cutaneous warts, and consideration of referral of family members for examination.

- Examination of the child for signs of physical injury. The majority of children who have been sexually abused have no genital or perineal injury on examination. ${ }^{7}$ The timing of the examination and nature of the abuse are critical factors.

- Examination to look for genital warts. The diagnosis of EGWs is usually a clinical one and should be differentiated from anatomically normal findings, such as micropapillomatosis labialis in females ${ }^{42}$ or pearly penile papules in males. Biopsy may occasionally be necessary for atypical or persistent lesions (such as bowenoid papulosis and giant condylomas). ${ }^{42}$ Colposcopic examination (a common practice in child sexual abuse clinics) has low specificity for the detection of HPV alone, in the absence of EGWs, as false positive findings can occur with other inflammatory conditions. ${ }^{42}$

- A physical examination to exclude other sexually transmitted infections $\left(\right.$ STIS) ${ }^{19}$ (prevalent in less than $4 \%$ of sexually abused children.) In many centres a nucleic acid amplification test (NAAT) for Chlamydia trachomatis via a first pass urine is advocated as the first test, although false positives may result and CDC guidelines recommend that a second NAAT test be performed to a different DNA target, for confirmation. ${ }^{43}$ Preliminary analyses of a CDC trial investigating the use of urine NAATs in children has shown $100 \%$ sensitivity compared to vaginal culture for $C$ trachomatis, suggesting that they may one day become a new forensic standard for diagnosis of bacterial STIs in children suspected of sexual abuse. ${ }^{44}$

- A behavioural and social assessment by skilled professionals.

- Suspected sexual abuse cases should be reported to appropriate authorities.

- Emotional support and education. Children with HPV may experience significant emotional distress, shame and fear, and should be positively assured that carriage of HPV is common, likely to be self-limiting, and unlikely to result in significant morbidity. Notions of self-blame should be dispelled.

- Follow up. Children with EGWs should be treated and followed up for signs of recurrence (which can occur in 20-30\%). Long term prospective studies are required to assess whether HPV DNA typing and earlier Pap screening could play a role to select a population of young women that may be at higher risk of developing early onset cervical cancer. UK guidelines do not support cervical screening in adolescence. ${ }^{45}$ 


\section{The case study reviewed}

Had the child contracted infection from the mother?

Perinatal infection via maternal HPV infection (asymptomatic carriage, dysplasia, or EGWs) is a possibility because of the child's age. If the child were older it is an unlikely cause of transmission. HPV does display latency however, and in some situations may declare itself clinically later than expected (for example during significant immunosuppression). Contraction of EGWs from maternal finger warts is a possibility, although of low risk probability.

Had the child contracted infection from the caregiver?

In the absence of finger warts, horizontal transmission from the carer (via innocent heteroinoculation, e.g. during toileting, or by sexually abusive genital fondling) is not a proven mode of transmission to date. Viral DNA from finger samples has been found in people with genital warts, although we are unaware if the presence of HPV DNA per se implies infectivity. Sexual transmission may well have occurred from penile-vulvo-vaginal contact.

Is it possible to compare viral types (DNA fingerprinting) to look for clonality?

HPV $6 / 11$ are relatively stable viruses and do not vary markedly between hosts. Due to the high prevalence of types 6 and 11 , if DNA fingerprinting were done on the warts from the carer and the child, the chances are that the viral types will be identical, irrespective of the source and/or mode of transmission and hence represent the likely circulating type for the area. Furthermore, a finding of identical viral types between the child and the carer would not indicate the mode of transmission.

Could the child have picked up the virus from fomites?

Transmission of HPV DNA to the external genital tract via fomites is a remote possibility, but not proven. Moreover, it is still unclear if this transmission route would be capable of inducing active infection and clinical HPV infection (such as EGWs).

Does this child have a high risk of developing cervical cancer?

EGWs are benign but are surrogate markers for the carriage of high risk subtypes in adults. The incidence of high risk HPVs and their potential oncogenic outcome when acquired before puberty is unknown. Young women with HPV tend to clear the virus. The child who has persistent chronic infection with high risk genotypes of HPV and who develops high grade dysplasia is at risk of developing cervical cancer, but whether this is greater risk at a younger age we do not know.

\section{Authors' affiliations}

Y Jayasinghe, Department of Paediatric \& Adolescent Gynaecology, Centre for Adolescent Health, Royal Children's Hospital, Melbourne, Australia

S M Garland, Department of Microbiology \& Infectious Diseases, Royal Women's Hospital, Victoria, Australia; Department of Obstetrics and Gynaecology, University of Melbourne, Australia

Competing interests: none

\section{REFERENCES}

1 Garland S. Human papillomavirus update with a particular focus on cervical disease. Pathology 2002;34:213-24.

2 Syrianen S. HPV infections in children. Papillomavirus Report 2003;14:93-110.

3 Armstrong DK, Handley JM. Anogenital warts in prepubertal children: pathogenesis, HPV typing and management. Int J STD AIDS 1997;8:78-81.

4 Moscicki A. Genital infections with human papillomavirus (HPV). Pediatr Infect Dis J 1998; 17:651-2.

5 Gibbs N. Anogenital papillomavirus infections in children. Curr Opin Pediatr 1998; 10:393-7.

6 Atabaki S, Paradise J. The medical evaluation of the sexually abused child: lessons learned from a decade of research. Pediatrics 1999;104:178-86.

7 Berenson AB, Chacko MR, Wiemann CM, et al. A case-control study of anatomic changes resulting from sexual abuse. Am J Obstet Gynecol 2000;182:820-34.

8 Sinclair K, Woods C, Kirse D, et al. Anogenital and respiratory tract human papillomavirus infections among children: age, gender, and potential transmission through sexual abuse. Pediatrics 2005;116:815-25.

9 Oriel JD. Natural history of genital warts. Brit J Vener Dis 1971;47:1-13

10 Hermonat $\mathbf{P}$, Kechlava S, Lowery C, et al. Trophoblasts are the preferential target for human papillomavirus infection in spontaneously aborted products of conception. Hum Pathol 1998;29:170-4.

11 Armbruster-Moraes E, loshimoto L, Leao E, et al. Presence of HPV DNA in amniotic fluid of pregnant women with cervical lesions. Gynecol Oncol 1994:54:152-8.

12 Tang C, Shermeta D, Wood D. Congenital condyloma acuminata. Am J Obstet Gynecol 1978;131:912-13.

13 Pao C, Lin S, Lin C, et al. Identification of human papillomavirus DNA sequences in peripheral blood mononuclear cells. Am J Clin Pathol 1991:95:540-6.

14 Tseng C, Lin C, Wang R, et al. Possible transplacental transmission of human papillomavirus. Am J Obstet Gynecol 1992;166:35-40.

15 Bodaghi S, Wood LV, Roby G, et al. Could human papillomaviruses be spread through blood? J Clin Microbiol 2005:43:5428-34.

16 Cason J, Kaye JN, Jewers RJ, et al. Perinatal infection and persistence of human papillomavirus type 16 \& 18 in infants. J Med Virol 1995;47:209-18.
17 Watts D, Koutsky L, Holmes K, et al. Low risk of perinatal transmission of human papillomavirus: results from a prospective cohort study. Am J Obstet Gynecol 1998; 178:365-73.

18 Thompson C, Macdonald M, Sutherland S. Family cluster of Chlamydia trachomatis infection. BMJ 2001;322:1473-4.

19 Hammerschlag M. Sexually transmitted diseases in sexually abused children: medical and legal implications. Sex Transm Infect 1998;74:167-74.

20 Powell J, Strauss S, Gray J, et al. Genital carriage of human papillomavirus (HPV) DNA in prepubertal girls with and without vulval disease. Pediatr Dermatol 2003;20:191-4.

21 Kashima HK, Shah K. Recurrent respiratory papillomatosis. Clinical overview and management principles. Obstet Gynecol Clin North Am 1987;14:581-8.

22 Cason J, Mant C. High-risk mucosal human papillomavirus infections during infancy and childhood. J Clin Virol 2005;32(suppl 1):52-8

23 De Villiers EM. Human pathogenic papillomavirus types: an update. Curr Top Microbiol Immunol 1994;186:1-12.

24 Bosch FX, Iffner T. The aetiology of cervical cancer. Sheffield, UK: NHS Cervical Screening Programme, 2005.

25 Pao C, Tsai P, Chang Y, et al. Possible non-sexual transmission of genital human papillomavirus infections in young women. Eur J Clin Microbiol Infect Dis 1993; 12:221-3.

26 Pacheco B, Di Paola G, Ribas J, et al. Vulvar infections caused by human papillomavirus in children and adolescents without sexual contact. Adolesc Pediatr Gynecol 1991;4:136-42.

27 Fairley CK, Chen S, Tabrizi S, et al. The absence of genital human papillomavirus DNA in virginal women. Int J STD AIDS 1992;3:414-17.

28 Rylander E, Ruusuvaara L, Wiksten MA, et al. The absence of vaginal human papillomavirus 16 DNA in women who have not experienced sexual intercourse. Obstet Gynecol 1994;83:735-7.

29 Anderson-Ellstrøm A, Hagmar BM, Johansson B, et al. Human papillomavirus deoxyribonucleic acid in cervix only detected in girls after coitus. Int J STD AIDS 1996;7:333-6.

30 Kjaer SK, Chackerian B, van den Brule AJC, et al. High-risk human papillomavirus is sexually transmitted: evidence from a follow-up study of virgins starting sexual activity (intercourse). Cancer Epidemiol Biomark Prev 2001; 10:101-6.

31 Snijders $\mathbf{P}$, van den Brule A, Meijer $C$. The clinical relevance of human papillomavirus testing: relationship between analytical and clinical sensitivity. J Pathol 2003;201:1-6.

32 Rice PS, Mant C, Cason J, et al. High prevalence of human papillomavirus type 16 infection among children. J Med Virol 2000;61:70-5.

33 Guido R. Guidelines for screening and treatment of cervical disease in the adolescent. J Pediatr Adolesc Gynecol 2004;17:303-11.

34 Gray S, Walzer T. New strategies for cervical cancer screening in adolescents. Curr Opin Pediatr 2004;16:344-9.

35 Gutman L, St Claire K, Everett VD. Cervico-vaginal and intra-anal human papillomavirus infection in young girls with external genital warts. J Infect Dis $1994 ; 170: 339-44$. 
36 Breneman D, Lucky A, Ostrow RS, et al. Bowenoid papulosis of the genitalia associated with human papillomavirus DNA type 16 in an infant with atopic dermatitis. Pediatr Dermatol 1985;2:297.

37 Brown D, Shew M, Qadadri B, et al. A longitudinal study of genital human papillomavirus infection in a cohort of closely followed adolescent women. $J$ Infect Dis 2005;191:182-92.

38 Winer R, Kiviat N, Hughes J, et al. Development and duration of human papillomavirus lesions after initial infection J Infect Dis 2005;191:731-8.

39 Woodman C, Collins S, Winter $\mathrm{H}$, et al. Natural history of cervical human papillomavirus infection in young women: a longitudinal cohort study. Lancet 2001;357:1831-6.

40 Mao C, Koutsky LA, Ault KA, et al. Effectiveness of human papillomavirus 16 virus like particle vaccine in preventing human papillomavirus 16-related cervical intraepithelial neoplasia 2-3: a randomized controlled trial. Obstet Gynecol 2006;107:18-27.
41 NHMRC. Screening to prevent cervical cancer: guidelines for management of asymptomatic women with screen detected abnormalities. National Health and Medical Research Council, June, 2005.

42 Von Krogh G, Lacey C, Gross G, et al. European course on HPV associated pathology: guidelines for primary care physicians for the diagnosis and management of anogenital warts. Sex Transm Infect 2000;76: 162-8.

43 Centers for Disease Control and Prevention. Sexual assault and STDs. Sexually transmitted diseases treatment guidelines. MMWR Recomm Rep 2002;51(RR-6):69-74.

44 Black CM, Driebe EM, Beck-Sague CM, et al. Urine testing for Chlamydia trachomatis and Neisseria gonorrhoeae in sexually abused children. Abstract American Society of Microbiology, 2005.

45 Health Service Cervical Screening Programme. 1987 www.cancerscreening.nhs.uk.

\section{ARCHIVIST}

\section{Atrial septostomy for pulmonary hypertension}

diopathic pulmonary arterial hypertension (IPAH) has a poor prognosis although there have been recent advances in medical treatment. Atrial septostomy has been used in adults with IPAH who have usually been critically ill with recurrent syncope or right heart failure. The procedure induces right to left shunting, thus decreasing the strain on the right side of the heart and maintaining left ventricular output. Improvements in survival and quality of life have been reported but at a cost of high perioperative risk. Experience in children has been limited but a series of 20 children has now been reported from the UK Service for Pulmonary Hypertension in London (A Micheletti and colleagues. Heart, published Online First 8 November 2005; 10.1136/hrt.2005.077669).

Nineteen children aged $0.25-17.0$ years at the time of septostomy had IPAH and one child aged 16.5 years had pulmonary hypertension secondary to HIV infection. All were severely symptomatic, 13 with recurrent syncope (one also with right heart failure) and seven with right heart failure without syncope. Medical treatments had included bosentan, iloprost, nifedipine, epoprostenol, and sildenafil.

The procedure was graded balloon septostomy alone in 8 cases, insertion of a fenestrated device in 7, blade septostomy plus graded balloon septostomy in 3, and blade septostomy alone in 2. The mean interval between diagnosis and septostomy was 2.5 years. A functional septal aperture was created in all patients except the one with pulmonary hypertension secondary to HIV infection. The procedure was complicated in four cases. One had arrhythmias and cardiac arrest just before the procedure, one had a cardiac arrest after receiving a fenestrated device, a third suffered hypoxaemia and acidosis after insertion of a fenestrated device, and a fourth (with HIV infection) suffered hypoxaemia during graded balloon septostomy. Mean systemic arterial oxygen saturation fell from $97.8 \%$ before septostomy to $90 \%$ afterwards. There were no early deaths. One child aged 3 months at the time of the procedure continued to have syncopal episodes and died aged 5 months. Another child who was 6 years old at the time of septostomy died after a major haemoptysis 5 years later. Two children needed repeat septostomies. Syncope was abolished in all cases apart from the infant who died. All 20 patients were discharged home, 15 on continuous epoprostenol infusion.

Atrial septostomy benefited these patients. The authors of this paper suggest that it should be done relatively early, before the patient deteriorates. 